International Journal of Gynecological Pathology:

January 2007 - Volume 26 - Issue 1 - pp 83-88

doi: 10.1097/01.pgp.0000225850.90115.bc

Pathology of the Lower Genital Tract

\title{
An Immunohistochemical Study of CD1a and CD83- Positive Infiltrating Dendritic Cell Density in Cervical Neoplasia
}

\author{
Hayati, Abdul Rahman M.B.B.S.; Zulkarnaen, Mohammad
}

\begin{abstract}
Cervical carcinoma is the second leading cancer in women in Malaysia, after breast cancer. Human papillomavirus (HPV) has been implicated in the development of dysplasia or cervical intraepithelial neoplasia and progression to squamous cell carcinoma. Because of the confinement of the human papillomavirus infection within the epithelial layer, the presence of dentritic cells or Langerhans cells in epithelial layer of the ectocervix is paramount in producing immune response. The mature dentritic cells express CD83 and high CD40/80/86, whereas the immature cells express CD1a and low CD40/80/86. By identifying CD1a and CD83, theoretically, both immature and mature dentritic cell populations can be studied. In view of the facts, we investigated the infiltrating cell density of mature and immature dentritic cells in cervical neoplasia.
\end{abstract}

C2007International Society of Gynecological Pathologists 